\title{
HLA-class I markers and multiple sclerosis susceptibility in the Italian population
}

\author{
L Bergamaschi $^{1}$, MA Leone ${ }^{2}$, ME Fasano ${ }^{3}$, FR Guerini ${ }^{4}$, D Ferrante ${ }^{5}$, E Bolognesi $^{1}$, N Barizzone ${ }^{1}$, \\ L Corrado $^{1}$, P Naldi ${ }^{2}$, C Agliardi ${ }^{4}$, E Dametto ${ }^{3}$, M Salvetti ${ }^{6}$, A Visconti ${ }^{6}$, D Galimberti ${ }^{7}$, E Scarpini ${ }^{7}$, \\ M Vercellino ${ }^{8}$, R Bergamaschi ${ }^{9}$, F Monaco ${ }^{10,11}$, D Caputo ${ }^{12}$, P Momigliano-Richiardi ${ }^{1}$ and S D' Alfonso ${ }^{1}$ \\ ${ }^{1}$ Department of Medical Sciences and Interdisciplinary Research Center of Autoimmune Diseases (IRCAD), University of Eastern \\ Piedmont, Novara, Italy; ${ }^{2}$ Department of Neurology, Ospedale Maggiore and IRCAD, Novara, Italy; ${ }^{3}$ Transplantation Immunology, San \\ Giovanni Battista Hospital, Turin, Italy; ${ }^{4}$ Laboratory of Molecular Medicine and Biotechnology, Don C Gnocchi Foundation IRCCS, \\ $S$ Maria Nascente, Milan, Italy; ${ }^{5}$ Department of Medical Sciences, Unit of Medical Statistics and Cancer Epidemiology, University \\ of Eastern Piedmont and CPO Piemonte, Novara, Italy; ${ }^{6}$ Neurology and Center for Experimental Neurological Therapy (CENTERS), \\ Università La Sapienza, Roma, Italy; ${ }^{7}$ Department of Neurological Sciences, Dino Ferrari Center, University of Milan, Fondazione \\ Ospedale Maggiore Policlinico, Milan, Italy; ${ }^{8}$ Department of Neurology, Ospedale San Giovanni Battista, Torino, Italy; ${ }^{9}$ Neurological \\ Institute C Mondino, IRCCS, Pavia, Italy; ${ }^{10}$ Department of Neurology, Ospedale Maggiore, Novara, Italy; ${ }^{11}$ Department of Clinical and \\ Experimental Medicine, University of Eastern Piedmont, Novara, Italy and ${ }^{12}$ Multiple Sclerosis Unit, Don C Gnocchi Foundation, \\ IRCCS, S Maria Nascente, Milan, Italy
}

Previous studies reported an association with multiple sclerosis (MS) of distinct HLA-class I markers, namely HLA-A*02, HLA-CW*05 and MOG-142L. In this work, we tested the association with MS of $A^{* 02}$ and CW*05 in 1273 Italian MS patients and 1075 matched controls, which were previously analyzed for MOG-142, and explored the relationship among these three markers in modulating MS risk. HLA-A*02 conferred a statistically robust MS protection (odds ratio, OR $=0.61 ; 95 \%$ confidence intervals, $\mathrm{Cl}=0.51-0.72, \mathrm{P}<10^{-9}$ ), which was independent of DRB1*15 and of any other DRB1* allele and remained similar after accounting for the other two analyzed class I markers. Conversely, the protective effect we previously observed for MOG$142 \mathrm{~L}$ was secondary to its linkage disequilibrium with $A^{*} 02$. C $w^{*} 05$ was not associated considering the whole sample, but its presence significantly enhanced the protection in the HLA-A*02-positive group, independently of DRB1: the OR conferred by $A^{*} 02$ in C $w^{*} 05-p o s i t i v e ~ i n d i v i d u a l s ~(0.22,95 \% ~ C l=0.13-0.38)$ was significantly lower than in $C w^{*} 05$-negative individuals (0.69, $95 \% \mathrm{Cl}=0.58-0.83)$ with a significant $\left(P=4.94 \times 10^{-5}\right)$ multiplicative interaction between the two markers. In the absence of $A^{*} 02, C w^{*} 05$ behaved as a risk factor, particularly in combination with $D R B 1^{*} 03(O R=3.89, P=0.0006)$, indicating that $C w^{*} 05$ might be a marker of protective or risk haplotypes, respectively.

Genes and Immunity (2010) 11, 173-180; doi:10.1038/gene.2009.101; published online 12 November 2009

Keywords: multiple sclerosis; HLA-class I markers; extended HLA haplotypes; Myelin Oligodendrocyte Glycoprotein; genetic association

\section{Introduction}

Multiple sclerosis (MS) is an autoimmune disease of the central nervous system characterized by disseminated and focal damage of myelin and axons, resulting in a disabling condition. ${ }^{1}$ The pathogenesis of MS is not understood yet, but several studies suggest an interaction of environmental and genetic factors. ${ }^{2}$ The genetic factor showing the strongest association with MS is

Correspondence: Dr S D'Alfonso, Department of Medical Sciences and Interdisciplinary Research Center of Autoimmune Disease (IRCAD), University of Eastern Piedmont, Via Solaroli 17, Novara 28100, Italy.

E-mail: dalfonso@med.unipmn.it

Received 13 April 2009; revised 22 September 2009; accepted 23 September 2009; published online 12 November 2009 localized in the human Major Histocompatibility complex (HLA) region on chromosome 6p21.3. The risk haplotype that has been identified for the Caucasian populations is HLA DRB1*1501-DQB1*0602 (also known as DR15 haplotype) in the HLA-class II region. In the Italian $^{3}$ as well as in other European populations, ${ }^{4-6}$ DR15 confers an odds ratio (OR) of about 3.

In recent years, several studies have searched for the presence of MS susceptibility factors in the HLA region with an effect independent of HLA-DRB1. No evidence of an HLA-A or -B association was found by Chao et al. ${ }^{7}$ in 294 Canadian families. Moreover, none of the 1068 single-nucleotide-polymorphisms (SNPs) from a highdensity panel spanning the entire HLA genomic region showed additional association in 1185 Canadian and Finnish families after conditioning for HLA-DRB1. ${ }^{8}$ Conversely, other studies have detected the effect at 
least of one class I genetic factor independently of $H L A$ DRB1. Brynedal et al. ${ }^{9}$ confirmed in a Nordic cohort of 1084 MS patients and 1347 controls the results of a previous study performed in a smaller Swedish panel, ${ }^{10}$ showing that HLA-A*02 is negatively associated with MS $\left(\mathrm{OR}=0.63, P=7 \times 10^{-12}\right)$. Yeo et al. ${ }^{11}$ analyzed over 1600 UK MS patients and 3600 controls and found that $H L A-C w^{*} 05$ exerts an MS-protective effect $(\mathrm{OR}=0.49$, $95 \%$ confidence intervals, $\mathrm{CI}=0.34-0.69, P=3.3 \times 10^{-5}$ ) after excluding all individuals carrying DRB1 alleles associated with MS. In a panel of 1124 Italian MS patients and 1136 controls, we found a significant association with the missense variant V142L (rs2857766) in the gene encoding the Myelin Oligodendrocyte Glycoprotein (MOG) mapping in the HLA-class I region, telomeric to $H L A-A(0.3 \mathrm{Mb})$ and $H L A-C(1.6 \mathrm{Mb}) .^{12}$ The $142 \mathrm{~L}$ allele $(M O G-142 L)$ conferred an OR $=0.70(95 \%$ C.I $=0.60-0.82)$ that remained similar after accounting for HLA-DRB1*15 carrier status. ${ }^{12}$ Burfoot et al..$^{13}$ showed similar data in a small Tasmanian population. Moreover, they reported a negative association with $H L A-A^{*} 02$, but they did not analyze if this variation was primarily associated or was dependent on MOG-142L. ${ }^{13}$

The relationship among the three reported HLA-class I markers, $A^{*} 02, C w^{*} 05, M O G-142 L$ and their association with MS has not been examined so far.

In this work, we tested the association of $A^{*} 02$ and $C w^{*} 05$ in a large cohort of Italian MS patients and controls, most of whom had been previously typed for MOG-142 polymorphism, and we explored the relationship among MOG-142L, HLA-A*02 and HLA-Cw*05 in modulating MS risk.

\section{Results}

Association with MS of MOG-142L, HLA-A*02 and $H L A-C w^{*} 05$ in the Italian population

The MS association of MOG-142L, HLA-A*02 and HLA$C w^{*} 05$ was tested in a case-control study (Table 1 ). The frequency of MOG-142L and HLA- $A^{*} 02$-positive individuals was significantly lower in cases than in controls, confirming the previously reported protective effect, $, 10,12,13$ whereas the association with $C w^{*} 05$ was not significant.
The comparison of genotype frequencies suggested a dominant-protective effect of MOG-142L and of HLA$A^{*} 02$, as their frequency was significantly decreased both among homozygous $(\mathrm{OR}=0.55,95 \% \mathrm{CI}=0.34-0.89$ for $142 \mathrm{~L}$ and $\mathrm{OR}=0.55,95 \% \mathrm{CI}=0.37-0.82$ for $\left.A^{*} 02\right)$ and heterozygous $(\mathrm{OR}=0.75,95 \% \mathrm{CI}=0.62-0.90$ for $142 \mathrm{~L}$ and $\mathrm{OR}=0.68,95 \% \mathrm{CI}=0.57-0.81$ for $\left.A^{*} 02\right)$ MS patients. The results did not change after adjustment for sex and were not different among male and female cases in a stratified analysis (data not shown). The results were not significantly different in relapsing remitting and primary progressive patients (data not shown).

To eliminate the possible confounding effect of linkage disequilibrium (LD) with $H L A-D R B 1^{*} 15$, we performed the same analysis in $D R B 1^{*} 15$-negative individuals. The OR values for the three tested markers remained substantially the same (Table 1).

The relative effect of the three tested HLA-class I markers on MS risk was evaluated by logistic regression analysis (Table 2). DRB1*15 was also included in the model. All individuals were categorized according to the presence or absence of each of the four considered alleles. The OR conferred by $A^{*} 02$ remained similar after accounting for each of the other markers included in the model and for all the markers together, showing that the protective effect was independent of any other analyzed HLA marker. Conversely, the protective effect of MOG$142 L$ was abolished by adjustment for $A^{*} 02$. The lack of a significant association with $C w^{*} 05$ and the significant risk effect of $D R B 1^{*} 15$ were similar with and without the adjustment for the remaining markers. This analysis clearly showed that the association with MOG-142L was not independent of $H L A-A^{*} 02$. This was also indicated by an analysis stratified for $H L A-A^{*} 02$ : MOG-142L was not significantly associated either in the $A^{*} 02$ positive $(\mathrm{OR}=0.9695 \% \mathrm{CI}=0.74-1.23, P=0.76)$ or in the $A^{*} 02$ negative $(\mathrm{OR}=0.80,95 \% \mathrm{CI}=0.60-1.07, P=0.13)$. These results were explained by the strong $\mathrm{LD}\left(D^{\prime}=0.59\right.$; $r^{2}=0.5$ ) between the two alleles both in the control and in the MS patient population (Table 3).

Considerable LD was also detected between $A^{*} 02$ and $C w^{*} 05$ in the control population (Table 3). Notably, it was completely absent in the MS patients. This difference was explained by the significantly decreased frequency of the

Table 1 Frequency of MOG-142L, HLA-A*02 and $H L A-C w^{*} 05$-positive individuals among MS patients and controls

\begin{tabular}{|c|c|c|c|c|c|}
\hline \multirow[t]{2}{*}{ Marker } & \multirow[t]{2}{*}{ Sample } & \multicolumn{2}{|c|}{ Frequencies } & \multirow[t]{2}{*}{ P-value } & \multirow[t]{2}{*}{ OR $(95 \% C I)$} \\
\hline & & Cases & Controls & & \\
\hline & Total & $\mathrm{N}=1273$ & $\mathrm{~N}=1075$ & & \\
\hline MOG-142L & & 0.285 & 0.364 & $4.93 \times 10^{-5}$ & $0.70(0.59-0.83)$ \\
\hline$H L A-A^{*} 02$ & & 0.331 & 0.448 & $5.28 \times 10^{-9}$ & $0.61(0.51-0.72)$ \\
\hline$H L A-C w^{*} 05$ & & 0.101 & 0.112 & NS & $0.85(0.65-1.10)$ \\
\hline & DR15 negative & $\mathrm{N}=883$ & $\mathrm{~N}=935$ & & \\
\hline MOG-142L & & 0.293 & 0.377 & $1.42 \times 10^{-4}$ & $0.68(0.56-0.84)$ \\
\hline$H L A-A^{*} 02$ & & 0.334 & 0.464 & $1.44 \times 10^{-8}$ & $0.58(0.48-0.70)$ \\
\hline$H L A-C w * 05$ & & 0.108 & 0.119 & NS & $0.89(0.66-1.21)$ \\
\hline
\end{tabular}

Abbreviations: CI, confidence intervals; MS, multiple sclerosis; OR, odds ratio; NS, not significant.

Genotype frequencies $(+/+,+/-,-/-)$ in the total MS patient sample vs total controls were $0.02,0.26,0.72$ vs $0.04,0.32,0.64$ for MOG-142L and $0.04,0.29,0.67$ vs $0.07,0.38,0.55$ for $H L A-A^{*} 02$. These frequencies did not deviate from Hardy Weinberg equilibrium either in MS patients or controls. 
Table 2 Logistic regression analysis of MOG-142L, HLA-A*02, HLA-Cw* 05 and DRB1*15 alleles

\begin{tabular}{|c|c|c|c|c|c|}
\hline \multirow[t]{2}{*}{ Markers } & \multicolumn{5}{|c|}{ OR $(95 \%$ CI $)$ adjusted values for: } \\
\hline & MOG-142L & $H L A-A^{*} 02$ & $H L A-C w^{*} 05$ & $D R B 1^{*} 15$ & All markers in the model \\
\hline MOG-142L & $0.70(0.59-0.83)$ & $\begin{array}{l}0.87(0.71-1.06) \\
P=0.1761\end{array}$ & $\begin{array}{l}0.70(0.59-0.83) \\
P=6.2 \times 10^{-5}\end{array}$ & $\begin{array}{l}0.73(0.61-0.87) \\
P=0.0004\end{array}$ & $0.90(0.73-1.10)$ \\
\hline$H L A-A^{*} 02$ & $\begin{array}{l}0.65(0.53-0.79) \\
P=1.0 \times 10^{-5}\end{array}$ & $0.61(0.51-0.72)$ & $\begin{array}{l}0.61(0.52-0.72) \\
P=8.2 \times 10^{-9}\end{array}$ & $\begin{array}{l}0.63(0.53-0.75) \\
P=1.0 \times 10^{-7}\end{array}$ & $0.66(0.54-0.81)$ \\
\hline$H L A-C w^{*} 05$ & $\begin{array}{l}0.87(0.67-1.13) \\
P=0.2970\end{array}$ & $\begin{array}{l}0.90(0.69-1.17) \\
P=0.4167\end{array}$ & $0.85(0.65-1.10)$ & $\begin{array}{l}0.88(0.67-1.14) \\
P=0.3348\end{array}$ & $0.92(0.71-1.21)$ \\
\hline$D R B 1^{*} 15$ & $\begin{array}{l}2.90(2.34-3.59) \\
P=2.8 \times 10^{-24}\end{array}$ & $\begin{array}{l}2.89(2.33-3.58) \\
P=6.3 \times 10^{-24}\end{array}$ & $\begin{array}{l}2.94(2.37-3.64) \\
P=4.6 \times 10^{-25}\end{array}$ & $2.95(2.38-3.65)$ & $2.87(2.32-3.56)$ \\
\hline
\end{tabular}

Abbreviations: CI, confidence intervals; OR, odds ratio.

Each marker in row is adjusted for markers in columns. In the last column the model includes all the markers.

The bolded diagonal values contain the crude values for each marker.

$P$-values are obtained from likelihood ratio test comparing the likelihood of the two-gene additive vs the single marker model considering as single marker the marker used for adjustment.

Table 3 Linkage disequilibria among the three considered HLA-class I markers and $D R B^{*} 15$ in the control and in the MS patient population

\begin{tabular}{|c|c|c|c|c|c|c|c|c|}
\hline & \multicolumn{4}{|c|}{ Controls } & \multicolumn{4}{|c|}{ MS patients } \\
\hline & MOG-142L & $A^{*} 02$ & $C w^{*} 05$ & $D R B 1^{*} 15$ & MOG-142L & $A^{*} 02$ & $C w^{*} 05$ & $D R B 1{ }^{*} 15$ \\
\hline MOG-142L & - & & & & - & & & \\
\hline$A^{*} 02$ & $0.587^{* * *}(0.469)$ & - & & & $0.588^{* * *}(0.510)$ & - & & \\
\hline$C w^{*} 05$ & $0.194 *(0.091)$ & $0.403^{* * *}(0.160)$ & - & & $0.002(0.001)$ & $-0.003(0.015)$ & - & \\
\hline$D R B 1 * 15$ & $-0.011^{* *}(0.074)$ & $-0.014^{* *}(0.082)$ & $-0.001(0.012)$ & - & $-0.006(0.028)$ & $-0.003(0.011)$ & $-0.003(0.031)$ & - \\
\hline
\end{tabular}

Abbreviation: MS, multiple sclerosis.

Numbers represent $\mathrm{D}^{\prime}$ (Lewontin's delta) and $r^{2}$ (in brackets) values.

${ }^{*} P<0.05,{ }^{* *} P<0.005$ and ${ }^{* * *} P<0.000001$.

Table 4 Phenotypic combinations of $H L A-A^{*} 02$ and $H L A-C w^{*} 05$ in MS patients and controls

\begin{tabular}{|c|c|c|c|c|c|c|}
\hline \multirow[t]{2}{*}{$A^{*} 02$} & \multirow[t]{2}{*}{$C w^{*} 05$} & \multirow[t]{2}{*}{ Sample } & \multicolumn{2}{|c|}{ Frequency } & \multirow[t]{2}{*}{ P-value } & \multirow[t]{2}{*}{ OR $(95 \% C I)$} \\
\hline & & & Cases & Controls & & \\
\hline & & Total & $\mathrm{N}=1273$ & $\mathrm{~N}=1075$ & & \\
\hline - & - & & 0.599 & 0.513 & $2.4 \times 10^{-5}$ & $1.42(1.21-1.68)$ \\
\hline+ & + & & 0.031 & 0.078 & $4.1 \times 10^{-7}$ & $0.38(0.26-0.56)$ \\
\hline+ & - & & 0.299 & 0.370 & $2.8 \times 10^{-4}$ & $0.73(0.61-0.86)$ \\
\hline- & + & & 0.070 & 0.039 & 0.001 & $1.85(1.27-2.69)$ \\
\hline & & DR15 negative & $\mathrm{N}=883$ & $\mathrm{~N}=935$ & & \\
\hline- & - & & 0.589 & 0.498 & $1.1 \times 10^{-4}$ & $1.44(1.20-1.73)$ \\
\hline+ & + & & 0.031 & 0.081 & $1.8 \times 10^{-6}$ & $0.36(0.23-0.56)$ \\
\hline+ & - & & 0.303 & 0.383 & $3.6 \times 10^{-4}$ & $0.70(0.58-0.85)$ \\
\hline- & + & & 0.077 & 0.038 & $2.4 \times 10^{-4}$ & $2.15(1.41-3.26)$ \\
\hline
\end{tabular}

Abbreviations: CI, confidence intervals; MS, multiple sclerosis; OR, odds ratio.

$A^{*} 02+, C w^{*} 05+$ phenotypic combination in the MS patient as opposed to the control population (Table 4). When analyzing in more detail the effect of the different phenotypic combinations of the $A^{*} 02$ and $C w^{*} 05$ alleles on MS risk (Table 4), it appeared that the $A^{*} 02+$, $C w^{*} 05+$ combination conferred an OR (0.38) significantly lower than that $(\mathrm{OR}=0.73)$ consequent to the presence of $A^{*} 02$ in the absence of $C w^{*} 05$. Conversely, in the absence of $H L A-A^{*} 02, C w^{*} 05$ showed a significant positive association with $\mathrm{MS}(\mathrm{OR}=1.85)$. The same results were seen when considering only $D R B 1^{*} 15$ negative individuals (Table 4 ).

Stratification for the $H L A-C w^{*} 05$ revealed a substantial difference in risk conferred by the $A^{*} 02$ among 
$C w^{*} 05$-positive individuals $(\mathrm{OR}=0.22,95 \% \mathrm{CI}=0.13-$ 0.38 ) and $C w^{*} 05$-negative individuals $(\mathrm{OR}=0.69,95 \%$ $\mathrm{CI}=0.58-0.83)$. When stratifying for $A^{*} 02, C w^{*} 05$ behaved as a protective factor among $A^{*} 02$-positive individuals $(\mathrm{OR}=0.50,95 \% \mathrm{CI}=0.33-0.74)$ and as a risk factor among $A^{*} 02$-negative individuals $(\mathrm{OR}=1.53,95 \%$ $\mathrm{CI}=1.04-2.24)$. The likelihood ratio test used to compare the full model (including the two markers and the interaction term) to the additive model (including only the two markers) was statistically significant $\left(P=4.9 \times 10^{-5}\right)$. Thus, although $H L A-C w^{*} 05$ and $A^{*} 02$ are not reciprocally confounders, as there was no difference between crude and adjusted ORs (Table 2), the stratification analysis showed a significant multiplicative interaction between the two markers.

From the analysis in the case-control population, it was not possible to distinguish whether the $A^{*} 02+$, $C w^{*} 05+$ individuals carried the two alleles in cis or in trans. We, therefore, analyzed the transmission of these two alleles in 201 family trios. No Mendelian errors were observed. Notably, out of 10 haplotypes that carried both $A^{*} 02$ and $C w^{*} 05$, none were transmitted to the MS patients (T:NT $=0: 10 ; P=0.002$ ). The only $A^{*} 02+$, $C w^{*} 05+$ MS patient present in this panel inherited the two alleles in trans. $A^{*} 02$-positive individuals of the family trios were sequenced to define the $A^{*} 02$ alleles at a higher resolution. In agreement with the literature (http://www.allelefrequencies.net/), in these samples, $A^{*} 0201$ was the most frequent $A^{*} 02$ allele $(90 \%)$ followed by $A^{*} 0205(8 \%)$ and $A^{*} 0217(2 \%) . A^{*} 0201$ was the most represented $A^{*} 02$ allele both in the $A^{*} 02+, C w^{*} 05+$ and in the $A^{*} 02+, C w^{*} 05-$ haplotypes. The same proportion of $A^{*} 02$ subtypes was seen among transmitted and nontransmitted haplotypes (data not shown).

The joint effect of class I $\left(A^{*} 02, C w^{*} 05\right)$ and class II $\left(D R B 1^{*} 15\right)$ markers on MS risk is reported in Figure 1. All the combinations of these three alleles showed a significantly increased risk relative to the $A^{*} 02+$, $C w^{*} 05+, D R B 1^{*} 15-$ phenotype, which is characterized both by the presence of the class I markers conferring the highest protection and by the absence of the class II allele conferring the highest risk. The three combinations carrying $D R B 1^{*} 15$ in the absence of the highly protective $A^{*} 02+, C w^{*} 05+$ phenotype (that is carrying only $A^{*} 02$ or only $C w^{*} 05$ or none of them) showed a similar eightfold increased risk. In the presence of both $A^{*} 02$ and $C w^{*} 05$, the $D R B 1^{*} 15$ risk approximately halved to 4.57 , although this difference was not statistically significant

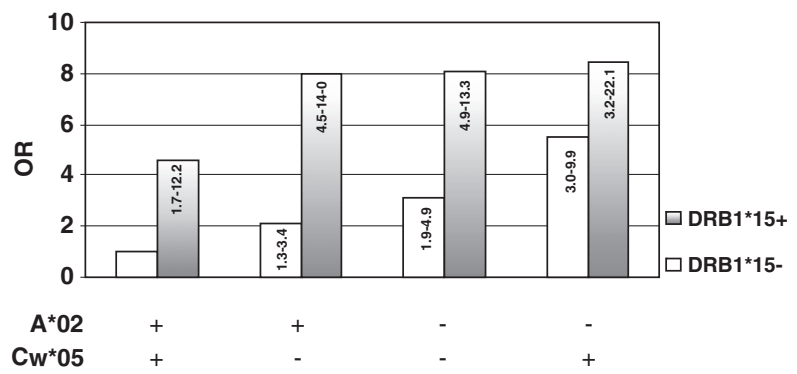

Figure 1 ORs for the different combinations of $A^{*} 02, C w^{*} 05$ and $D R B 1 * 15$ alleles. The combination carrying the HLA-class I alleles conferring the highest protection $\left(A^{*} 02+, C w^{*} 05+\right)$ and without the class II risk allele $\left(D R B 1^{*} 15-\right)$ was used as a reference $(\mathrm{OR}=1)$ for the calculation of ORs. Figures in each bar correspond to the $95 \% \mathrm{CI}$. owing to the low frequency of this phenotype $(N=13 \mathrm{MS}$ patients and 8 controls). In the absence of $D R B 1^{*} 15$, a differently increased risk was observed according to the presence of only $A^{*} 02(\mathrm{OR}=2.11)$ or only $C w^{*} 05$ $(\mathrm{OR}=5.47)$ or none of them $(\mathrm{OR}=3.14)$.

\section{Stratification for DRB1 alleles}

To test whether the detected associations were consequent on LD with $D R B 1$ alleles different from $D R B 1^{*} 15$, we evaluated the relationship of $H L A-A^{*} 02, C w^{*} 05$ and their combinations with the DRB1 locus in a random subset of 562 MS cases and 888 controls fully typed for $D R B 1$ at low resolution. In this subgroup, $D R B 1^{*} 15$ was positively $\mathrm{MS}$ associated with $\mathrm{OR}=2.86(95 \% \mathrm{CI}=2.14-$ 3.83; $\left.P=8.4 \times 10^{-14}\right)$. When considering $D R B 1^{*} 15$-negative individuals, there was an additional significantly positive association with $D R B 1^{*} 04 \quad(\mathrm{OR}=1.88,95 \%$ $\left.\mathrm{CI}=1.34-2.63, P=1.6 \times 10^{-4}\right)$ and a negative association with $D R B 1 * 07$ of borderline significance $(\mathrm{OR}=0.69,95 \%$ $\mathrm{CI}=0.49-0.98, P=0.036)$. At variance with other studies, $D R B 1^{*} 03$ was not associated $(\mathrm{OR}=1.0,95 \% \mathrm{CI}=0.72-$ 1.39). A modest global LD was detected between HLA$A^{*} 02$ and DRB1 (Global $D^{\prime}=0.149$, Cramer's $V=0.176$ ) and between $H L A-C w^{*} 05$ and DRB1 (Global $D^{\prime}=0.280$, Cramer's $V=0.194)$. The association with $A^{*} 02$ remained significant after conditioning on the DRB1 locus by the COCAPHASE program (unconditioned $P=1.9 \times 10^{-5}$; conditioned $\left.P=2.2 \times 10^{-4}\right)$, thus showing that it is independent of DRB1. $C w^{*} 05$ was not significantly associated (unconditioned $P=0.09$; conditioned $P=0.19)$. The OR conferred by $H L A-A^{*} 02$ did not substantially change after conditioning for each $D R B 1$ allele separately (data not shown). In addition, Cw*05 showed very similar results after conditioning for each $D R B 1$ allele with the notable exception of $D R B 1^{*} 03$ : a protective effect with borderline significance was evidenced for $H L A-C w^{*} 05$ when considering only $D R B 1^{*} 03$ negative individuals $(\mathrm{OR}=0.58,95 \% \mathrm{CI}=0.36-0.92$; $P=0.02)$. When the same procedure was applied to the different $A^{*} 02, C w^{*} 05$ combinations, there was no substantial difference after conditioning for each DRB1 allele separately, again with the exception of $D R B 1^{*} 03$ (Table 5). Interestingly, the $A^{*} 02$ negative, $C w^{*} 05$ positive combination was significantly increased among $D R B 1^{*} 03$-positive individuals $(\mathrm{OR}=3.89)$, whereas it was not significantly associated with MS among $D R B 1^{*} 03$-negative individuals $(\mathrm{OR}=0.96)$. The $A^{*} 02$ positive, $C w^{*} 05$ positive combination remained significantly protective both in $D R B 1^{*} 03$ positive $(\mathrm{OR}=0.28)$ and negative $(\mathrm{OR}=0.39)$ individuals. Thus, the presence of $C w^{*} 05$ was significantly protective in combination with $A^{*} 02$, independently of $D R B 1$, whereas in the absence of $A^{*} 02$, it behaved as a risk marker in combination with $D R B 1^{*} 03$ (Table 5).

\section{Discussion}

This work stems from previous studies undertaken by our group and others, each reporting an MS association with distinct HLA-class I markers, namely $H L A-A^{*} 02,{ }^{9,13}$ $H L A-C w^{*} 05^{11}$ and MOG-142L. ${ }^{12}$ We here analyze the relationship among these three markers for MS susceptibility. 
Table 5 Distribution of the phenotypic combinations of $H L A-A^{*} 02$ and $H L A-C w^{*} 05$ in MS patients and controls among $D R B 1^{*} 03$-positive and $D R B 1^{*} 03$-negative individuals

\begin{tabular}{|c|c|c|c|c|c|c|c|}
\hline & $A^{*} 02$ & $C w^{*} 05$ & $\begin{array}{l}\text { Cases } \\
\text { N (\%) }\end{array}$ & $\begin{array}{c}\text { Controls } \\
N(\%)\end{array}$ & $O R$ & $95 \% \mathrm{CI}$ & P-value \\
\hline \multicolumn{8}{|l|}{$D R B 1^{*} 03+$} \\
\hline & - & + & $20(0.19)$ & $10(0.06)$ & 3.89 & $1.74-8.66$ & 0.0006 \\
\hline & - & - & $57(0.54)$ & $82(0.46)$ & 1.34 & $0.83-2.18$ & NS \\
\hline & + & + & $5(0.05)$ & $26(0.15)$ & 0.28 & $0.11-0.77$ & 0.006 \\
\hline \multirow{2}{*}{\multicolumn{8}{|c|}{$D R B 1^{*} 03-$}} \\
\hline & - & + & $16(0.04)$ & $26(0.04)$ & 0.96 & $0.51-1.81$ & NS \\
\hline & - & - & $274(0.60)$ & $360(0.51)$ & $\begin{array}{l}0.40 \\
1.46\end{array}$ & $1.15-1.86$ & 0.002 \\
\hline & + & + & $13(0.03)$ & $49(0.07)$ & 0.39 & $0.21-0.74$ & 0.002 \\
\hline & + & - & $153(0.34)$ & $276(0.39)$ & 0.79 & $0.62-1.01$ & NS \\
\hline
\end{tabular}

Abbreviations: CI, confidence intervals; MS, multiple sclerosis; OR, odds ratio; NS, not significant.

We confirmed a strong protective effect of $H L A-A^{*} 02$, which was independent of $D R B 1^{*} 15$ as well as of any other DRB1 allele. This association has now been detected, with similar ORs, in three populations with a different genetic background, respectively, from Italy (this paper), Sweden ${ }^{9}$ and Tasmania ${ }^{13}$ based on a total of over 2600 MS patients and 2700 controls. These data concur to demonstrate a role in MS of this allele (or of a variation in $\mathrm{LD}$ with it). Conversely, Chao et al..$^{7}$ failed to observe a transmission distortion of the HLA- $A^{*} 02$ allele in a large family sample from Canada and no SNPs in the $H L A-A$ region showed association with MS independently of HLA-class II loci in Canadian and Finnish families. ${ }^{8}$ As both these studies were based on an intrafamilial association method, an obvious explanation of the discrepancy could be a stratification problem related to the case-control approach used in the present as well as the other two studies.9.,13 However, as a significant $H L A-A^{*} 02$ association was seen in independent studies and in three different populations, this simple explanation seems unlikely and further studies are needed to address this point.

The $H L A-A^{*} 02$ protection remained similar after accounting for the other two analyzed class I markers. Conversely, the protective effect of MOG-142L was secondary to its high linkage disequilibrium with $H L A-A^{*} 02$. This excluded a direct role of the missense V142L variation in the MOG gene, a previously suggested strong MS susceptibility candidate (reviewed in D'Alfonso et al..$^{12}$ ).

In this study, $C w^{*} 05$ was not associated with MS either in the whole sample or in DRB1*15-negative individuals. However, when stratifying the results separately for all DRB1 alleles, $C w^{*} 05$ conferred a significant protection for MS in the subgroup of individuals negative for $D R B 1^{*} 03$. This is partially in line with the data reported by Yeo et al. ${ }^{11}$ who observed a protective effect of $C w^{*} 05$ in the whole sample as well as in individuals negative for $D R B 1 * 03$ and other MS-associated DRB1 alleles (DRB1*15, DRB1*0103). Conversely, in DRB1*03-positive individuals, and in the absence of $A^{*} 02, C w^{*} 05$ was a risk marker and conferred a risk similar to $D R B 1^{*} 15$ $(\mathrm{OR}=3.89, P=0.0006)$. Moreover, $C w^{*} 05$ significantly enhanced the protection effect of $H L A-A^{*} 02$ : the OR conferred by $A^{*} 02$ among $C w^{*} 05$-positive individuals $(\mathrm{OR}=0.22,95 \% \mathrm{CI}=0.13-0.38)$ was about $1 / 3$ smaller than among $C w^{*} 05$-negative individuals $(\mathrm{OR}=0.69,95 \%$
$\mathrm{CI}=0.58-0.83)$. Thus, although $C w^{*} 05$ itself was not significantly associated to MS, it behaved as a modifier of the $H L A-A^{*} 02$-mediated protection. The protective effect of the $C w^{*} 05-A^{*} 02$ combination was independent of the presence of $D R B 1^{*} 03$ (Table 5).

These data suggest that the association of $C w^{*} 05$ with MS varies according to its haplotypic context. $C w^{*} 05$ is a marker of three ancestral or conserved extended HLA haplotypes, ${ }^{14-16}$ one of which carries $D R B 1^{*} 03$ and is negative for $A^{*} 02$, namely $18.2\left[A^{*} 30\right.$, $\left.C w^{*} 05, B^{*} 18, D R B 1^{*} 03\right]$, and the other two carry $A^{*} 02$, but not $D R B 1^{*} 03$, namely 44.1 [A*0201,Cw*0501, $B^{*} 4402$, $\left.D R B 1^{*} 0401\right]$ and $18.3 \quad\left[A^{*} 0201, C w^{*} 0501, B^{*} 1801\right.$, DRB1*1102].

The 18.2 HLA-extended haplotype is typical of the Sardinian population and, to a lesser extent, of other Mediterranean populations. Conversely, in the populations of northern European origin, this DR3 haplotype is very rare and the majority of $D R B 1^{*} 03$-positive individuals carry the $8.1\left[A 1, C w^{*} 07, B^{*} 08, D R B 1^{*} 03\right]$ extended haplotype. The positive association of the $A^{*} 02-$, $C w^{*} 05+, D R B 1^{*} 03+$ combination observed in this study may reflect the effect of the 18.2 haplotype. It is tempting to speculate that the strong positive association of MS with $D R B 1^{*} 03$ observed in Sardinia is related to the 18.2 haplotype and not only to $D R B 1^{*} 03$ itself.

As stated above, the $A^{*} 02-C w^{*} 05$ combination is carried by two HLA ancestral haplotypes (44.1 and 18.3). However, as the individuals included in this study were not typed for HLA-B and other HLA markers, from our data it is not possible to conclude whether the enhanced MS-protective effect of the $A^{*} 02-C w^{*} 05$ combination is due to an haplotype effect (that is the presence of a primarily associated protective factor carried by the extended haplotype marked by $A^{*} 02$ and $C w^{*} 05$ ) or to a direct interactive role of the two markers.

The evidence in favor of an haplotype effect is as follows: (i) $C w^{*} 05$ was not associated with MS in the absence of $A^{*} 02$. Actually, $C w^{*} 05$ behaved as a protective factor among $A^{*} 02$-positive individuals ( $\mathrm{OR}=0.50,95 \%$ CI $0.33-0.74)$ and as a risk factor among $A^{*} 02$-negative individuals $(\mathrm{OR}=1.53,95 \% \mathrm{CI} 1.04-2.24)$. (ii) In family trios, out of 10 haplotypes that carried both $A^{*} 02$ and $C w^{*} 05$, none were transmitted to the MS patients (T:NT $=0: 10 ; P=0.002$ ). The only $A^{*} 02+, C w^{*} 05+\mathrm{MS}$ patient present in this panel inherited the two alleles in trans. (iii) Each of the $A^{*} 02, B^{*} 44$ and $C w^{*} 05$ alleles 
(characterizing the 44.1 ancestral haplotype) was significantly decreased in the study of Yeo et al. ${ }^{11}$ However, the association with the $A^{*} 02-B^{*} 44-C w^{*} 05$ haplotypic combination was not investigated by the authors.

On the other hand, evidence reported in this study and from work undertaken using an experimental animal model ${ }^{17}$ points to a direct role of $A^{*} 02$. In our study, $\mathrm{A}^{* 02}$ was also significantly protective in the absence of $\mathrm{Cw}^{*} 05$ $\left(\mathrm{OR}=0.73, P<10^{-4}\right)$. Thus, an effect of $A^{*} 02$ alone cannot be excluded. Moreover, Friese et al. ${ }^{17}$ recently reported that the MS-like disease developed by double transgenic mice expressing both a human HLA-class I allele $\left(\mathrm{A}^{*} 03\right)$ and a human myelin-specific autoreactive T-cell receptor is completely prevented by further adding an HLA$A^{*} 0201$ transgene. Thus, $\mathrm{A}^{*} 02$, which protects against MS in human populations, also prevents an MS-like disease in transgenic humanized mice. This protection resulted from thymic deletion of autoreactive $\mathrm{T}$ cells, which greatly reduced their number in the periphery. ${ }^{17}$

Both $H L A-A$ and $-C$ genes are interesting candidates for a direct role in MS pathogenesis, as their encoded molecules present antigenic peptides and interact with NK receptors. ${ }^{18,19}$ However, several other genes in this region might be primarily associated with MS. The $1.3 \mathrm{Mb}$ interval within HLA-A and -C genes contains 46 genes $(24 \%$ of which encoding molecules involved in immune functions) and about 4000 SNPs, according to the recently reported sequence of eight HLA ancestral haplotypes ${ }^{20}$ and HapMap data (http://www.hapmap. org/). A genome-wide association study ${ }^{21}$ in about 1000 US MS patients and 1000 matched controls identified several MS-associated SNPs in the HLA-class I region after conditioning for HLA-DRB1*15. Among these, the top signals were localized around members of the tripartite motif (TRIM) gene family, mapping about $200 \mathrm{~kb}$ centromeric to HLA-A. Although their function is unknown, the presence of a RING domain suggests DNA-binding activity. ${ }^{21}$

In conclusion, this study provides additional supportive evidence indicating that the HLA-class I region does indeed exert an additional influence on the risk of MS, analogous to that reported for other autoimmune diseases. ${ }^{22-24}$ Moreover, it identifies haplospecific markers conferring a high MS protection. Notably, although in general DRB1*15-negative individuals have an about threefold lower MS risk relative to DRB1*15-positive individuals, their risk was significantly decreased (about eightfold) if they also carried $\mathrm{A}^{*} 02$ and $\mathrm{Cw}^{*} 05$ (Figure 1). The highly protective $\mathrm{A}^{*} 02-\mathrm{Cw}^{*} 05$ combination is rare (0.078 phenotypic frequency in our controls, 0.025 haplotype frequency in our family trios) and, therefore, unless it tags a primary factor with higher frequency, it confers a modest modification of the HLA attributable MS risk in the population. In any case, the identification of the mechanism mediating the protective effect might throw new light on MS pathogenesis.

\section{Materials and methods}

\section{Subjects}

A total of 1273 Italian MS patients (female:male ratio 2:1) diagnosed according to McDonald et al., ${ }^{25}$ were genotyped for the three HLA-class I markers considered in this study and for DRB1*15. For 201 of these, a DNA sample of the parents was also available (family trios). The mean age of the MS patients at disease onset was $31 \pm 10.16$ years, the mean age at time of analysis was $40 \pm 15.52$ years and the mean disease duration was $12 \pm 9.31$ years. Eighty-four percent of the patients were affected by the relapsing remitting, $7 \%$ by the secondary progressive and $9 \%$ by the primary progressive form of the disease, defined according to Lublin and Reingold. ${ }^{26}$ MS patients with Sardinian ancestors were excluded to avoid the introduction of confounding sources of heterogeneity. Enrolment of the MS patients followed their informed consent. The study was approved by the Ethical Committees of the collaborating clinical centers.

Controls included 1075 Italian individuals (medical students, university and hospital staff, blood donors; female:male ratio 1:1.1) matched for age and regional origin with the MS patients and also typed for all considered markers.

MS patients $(82 \%)$ and controls $(30 \%)$ in part overlap with those included in a previous paper ${ }^{12}$ and were selected for inclusion on the basis of the availability of DNA and of HLA genotypes.

\section{MOG-V142L typing}

The newly included samples were typed using a pre-designed TaqMan SNP Genotyping Assay (probe code: C_25474376_10). Reactions were performed according to the manufacturer's protocol using $25 \mathrm{ng}$ of DNA. The PCR reaction was set up on a 7000 Applied Biosystems instrument. Genotypes were detected using the 7000 System Software (Applied Biosystems, Foster City, CA, USA).

A sub-sample of 60 individuals were typed both with this method and with the method used in the previous paper. $^{12}$ The results were consistent for all tested samples.

\section{HLA- $A^{*} 02$ typing}

$H L A-A$ exon 2 was amplified using a specific couple of primers (Forward: 5'-CGACGCCGCGAGCCAGARGAT-3', Reverse: 5'-GGCCCGTCCGTGGGGGATGA-3'). The PCR product $(213 \mathrm{bp})$ was digested using the restriction enzyme Kpn2 I. This enzyme recognizes and cuts the rs3173427-T sequence that is specific of $H L A-A^{*} 02$. By this approach, it was possible to distinguish $H L A-A^{*} 02$ homozygotes (displaying two fragments of 159 and $54 \mathrm{bp}$ ) from heterozygotes (displaying three fragments of 159,54 and $213 \mathrm{bp}$ ). The digestion was performed at $55^{\circ} \mathrm{C}$ for $4 \mathrm{~h}$, and then the enzyme was inactivated at $80^{\circ} \mathrm{C}$ for $20 \mathrm{~min}$.

$H L A-A^{*} 02$-positive individuals of family trios were also analyzed by sequencing exon 2 and exon 3 to define $H L A-A^{*} 02$ alleles at a higher resolution. Exon 2 and 3 were amplified together in the same fragment (Forward: 5'-CGACGCCGCGAGCCAGARGAT-3', Reverse: 5'-AA CGGGAAGGAGACGCTGC- $3^{\prime}$ ). The reaction mix was

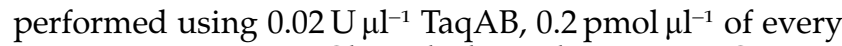
primers, $1.75 \mathrm{~mm} \mathrm{MgCl}_{2}$ and glycerol to $7.4 \%$. PCR was made at $60{ }^{\circ} \mathrm{C}$ annealing temperature for 35 cycles. PCR products were sequenced using nested primers: $5^{\prime}$ GGCCCGTCCGTGGGGGATGA-3 $3^{\prime}$ for exon 2 and $5^{\prime}$ TCAGTTTAGGCCAAAAATCC-3' for exon 3. Sequences were analyzed with the automatic sequencer Applied Biosystems (ABI) 3100. 
HLA-Cw*05 allele-specific PCR

All the samples were typed for $H L A-C w^{*} 05$ by an allelespecific PCR after the conditions of the 12th International Histocompatibility Workshop. ${ }^{27}$ In detail, specific primers pairs were used to amplify in the same tube HLA$\mathrm{Cw}^{*} 05$ (Forward: 5'-CCGAGTGAACCTGCGGAAA-3', Reverse: 5'-CGCGCGCTGCAGCGTCTT-3') and a 796 bp internal control fragment (Forward: 5'-TGCCAAGTGGA GCACCCAA-3', Reverse: 5'-GCATCTTGCTCTGTGCAG

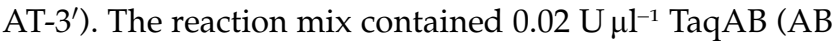
Analitica), $1 \mathrm{pmol} \mu \mathrm{l}^{-1}$ of $H L A-C w^{*} 05$-specific primers, $0.33 \mathrm{pmol}^{\mathrm{m}} \mathrm{l}^{-1}$ of internal control primers and $2 \mathrm{mM}$ of $\mathrm{MgCl}_{2}$. By this approach, it was possible to specifically identify all the samples positive for $H L A-C w^{*} 05$, but not to distinguish between $\mathrm{Cw}^{*} 05$ homozygotes and heterozygotes.

\section{DRB1 locus analysis}

For 562 MS cases and 888 controls, a complete lowresolution $D R B 1$ typing was already available. DRB1 alleles were typed by the DR low-resolution PCR-SSP (Sequence Specific Primer amplification) kit (Dynal or BAG, Formedic, Milan, Italy).

The remaining MS patients and controls were typed only for $D R B 1^{*} 15$ by an allele-specific PCR (Forward primer: 5'-CCTGTGGCAGCCTAAGAGG-3', Reverse primer: 5'-CCGCGCCTGCTCCAGGAT-3') with an internal control fragment (Forward: 5'-TGTTCTGTATTTGTGTTG TCTGATG-3', Reverse: 5'-GTGCTCAGAGAGGCAAGG TT- $\left.3^{\prime}\right)$. The reaction mix contained $0.02 \mathrm{U}_{\mu \mathrm{l}^{-1}}$ TaqAB

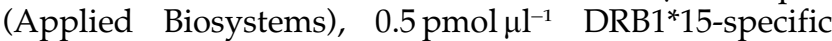
primers, $0.25 \mathrm{pmol}^{-1} \mathrm{l}^{-1}$ of internal control primers and $1.5 \mathrm{~mm}$ of $\mathrm{MgCl}_{2}$. By this approach, it was possible to specifically identify all the samples positive for DRB $1 * 15$, but not to distinguish between DRB1*15 homozygotes and heterozygotes.

\section{Quality control of allele-specific HLA typing}

The genotype methods used to type $\mathrm{A}^{*} 02, \mathrm{Cw}^{*} 05$ and DRB1*$^{*} 15$ alleles were validated by typing 51 HLA homozygous typing cell lines from the reference panel of the 12th International Histocompatibility Workshop ${ }^{27}$ and 55 individuals previously typed with a commercial kit.

\section{Statistical analysis}

Unconditional logistical regression was carried out to determine the effect of the considered markers on MS susceptibility. The association of each polymorphism with the disease was measured by the OR and its 95\% CI. Reported $P$-values were not corrected for the number of comparisons.

The potential confounding variables were assessed individually by comparing the log-likelihood ratios derived from a model with and without the variable. This analysis was set up using multivariate models using the four considered markers. All analyses were adjusted for sex. The different models were compared by the likelihood ratio test.

The interaction (modification) effect was assessed by comparing ORs across levels of potential modifying variables. Inclusion of appropriate interaction terms in the logistic regression model was used to assess the statistical significance of the interactions. For each marker, the potential effect modification by sex variable was also tested.

The main-effects test of the COCAPHASE program, part of the UNPHASED suite, ${ }^{28}$ was used for conditional analysis on DRB1. This program provides association tests conditioning on additional loci, which may already be associated and in linkage disequilibrium with the test loci. The EM algorithm is used to obtain maximiumlikelihood estimates of haplotypes.

LD were calculated from phenotypes according to Mattiuz et al. ${ }^{29}$ Estimates for Global D' and Cramer's V (measures of LD between multiallelic loci) were calculated using the COCAPHASE program. ${ }^{28}$

\section{Conflict of interest}

The authors declare no conflict of interest.

\section{Acknowledgements}

This work was supported by the Italian Foundation for Multiple Sclerosis (FISM grants 2001/R/44, 2002/R/40 and 2005/R/10, 2008/R/11); CARIPLO Foundation, Regione Piemonte Ricerca Sanitaria Finalizzata (grants 2003, 2004, 2007, 2008), Regione Piemonte CIPE 2004, Ministry of Health (ricerca finalizzata grant 2004.80), Eastern Piedmont University (grants to SD and PMR). Compagnia di San Paolo (Turin), Fondazione CRT (Turin). DG and ES were supported by Fondazione Monzino, Ospedale Maggiore Policlinico and Ing. Cesare Cusan. NB was supported by a fellowship from FISM (2003/B/2). LB is a 'Biotechnology for Health' PhD student supported by a $\mathrm{PhD}$ Lagrange Fellowship. We are grateful to the patients and their parents and also to Professor Corrado Magnani for helpful discussion and to Dr Roberto Tosi for critical suggestions.

\section{References}

1 Compston A, Coles A. Multiple sclerosis. Lancet 2008; 372: 1502-1517.

2 Ebers GC. Environmental factors and multiple sclerosis. Lancet Neurol 2008; 7: 268-277.

3 Ballerini C, Guerini FR, Rombolà G, Rosati E, Massacesi L, Ferrante $\mathrm{P}$ et al. HLA-multiple sclerosis association in continental Italy and correlation with disease prevalence in Europe. J Neuroimmunol 2004; 150: 178-185.

4 Fogdell A, Hillert J, Sachs C, Olerup O. The multiple sclerosisand narcolepsy-associated HLA class II Haplotype incluse DRB5*0101 allele. Tissue Antigens 1995; 46: 333-336.

5 Olerup O, Hillert J. HLA class II-associated genetic susceptibility in multiple sclerosis: a critical evaluation. Tissue Antigens 1991; 38: 1-15.

6 Barcellos LF, Sawcer S, Ramsay PP, Baranzini SE, Thomson G, Briggs $\mathrm{F}$ et al. Heterogeneity at the HLA-DRB1 locus and risk for multiple sclerosis. Hum Mol Genet 2006; 15: 2813-2824.

7 Chao MJ, Barnardo MC, Lui GZ, Lincoln MR, Ramagopalan SV, Herrera BM et al. Transmission of class I/II multi-locus MHC haplotypes and multiple sclerosis susceptibility: accounting for linkage disequilibrium. Hum Mol Genet 2007; 16: 1951-1958.

8 Lincoln MR, Montpetit A, Cader MZ, Saarela J, Dyment DA, Tiislar $\mathrm{M}$ et al. A predominant role for the HLA class II region 
in the association of the MHC region with multiple sclerosis. Nat Genet 2005; 37: 1108-1112.

9 Brynedal B, Duvefelt K, Jonasdottir G, Roos IM, Akesson E, Palmgren $\mathrm{J}$ et al. HLA-A confers an HLA-DRB1 independent influence on the risk of multiple sclerosis. PLoS One 2007; 7: e664.

10 Fogdell-Hahn A, Ligers A, Gronning M, Hillert J, Olerup O. Multiple sclerosis: a modifying influence of HLA class I genes in an HLA class II associated autoimmune disease. Tissue Antigens 2000; 55: 140-148.

11 Yeo TW, De Jager PL, Gregory SG, Barcellos LF, Walton A, Goris A et al. A second major histocompatibility complex susceptibility locus for multiple sclerosis. Ann Neurol 2007; 61: 228-236.

12 D'Alfonso S, Bolognesi E, Guerini FR, Barizzone N, Bocca S, Ferrante D et al. A sequence variation in the MOG gene is involved in multiple sclerosis susceptibility in Italy. Genes Immun 2008; 9: 7-15.

13 Burfoot RK, Jensen CJ, Field J, Stankovich J, Varney MD, Johnson LJ et al. SNP mapping and candidate gene sequencing in the class I region of the HLA complex: searching for multiple sclerosis susceptibility genes in Tasmanians. Tissue Antigens 2008; 71: 42-50.

14 Cattley SK, Williamson JF, Tay GK, Martinez OP, Gaudieri S, Dawkins RL. Further characterization of MHC haplotypes demonstrates conservation telomeric of HLA-A: update of the 4AOH and 10IHW cell panels. Eur J Immunogenet 2000; 27: 397-426.

15 Alper CA, Larsen CE, Dubey DP, Awdeh ZL, Fici DA, Yunis EJ. The haplotype structure of the human major histocompatibility complex. Hum Immunol 2006; 67: 73-84.

16 Dorak MT, Shao W, Machulla HK, Lobashevsky ES, Tang J, Park MH et al. Conserved extended haplotypes of the major histocompatibility complex: further characterization. Genes Immun 2006; 7: 450-467.

17 Friese MA, Jakobsen KB, Friis L, Etzensperger R, Craner MJ, McMahon RM et al. Opposing effects of HLA class I molecules in tuning autoreactive CD8+ T cells in multiple sclerosis. Nat Med 2008; 14: 1227-1235.

18 Moretta L, Moretta A. Killer immunoglobulin-like receptors. Curr Opin Immunol 2004; 16: 626-633.

19 Thananchai H, Gillespie G, Martin MP, Bashirova A, Yawata $\mathrm{N}$, Yawata $\mathrm{M}$ et al. Cutting edge: allele-specific and peptidedependent interactions between KIR3DL1 and HLA-A and HLA-B. J Immunol 2007; 178: 33-37.
20 Horton R, Gibson R, Coggill P, Miretti M, Allcock RJ, Almeida $\mathrm{J}$ et al. Variation analysis and gene annotation of eight MHC haplotypes: the MHC Haplotype Project. Immunogenetics 2008; 60: 1-18.

21 Baranzini SE, Wang J, Gibson RA, Galwey N, Naegelin Y, Barkhof $\mathrm{F}$ et al Genome-wide association analysis of susceptibility and clinical phenotype in multiple sclerosis. Hum Mol Genet 2009; 18: 767-778.

22 Howson JM, Walker NM, Clayton D, Todd JA, Diabetes Genetics Consortium. Confirmation of HLA class II independent type 1 diabetes associations in the major histocompatibility complex including HLA-B and HLA-A. Diabetes Obes Metab 2009; 11(Suppl 1): 31-45.

23 Eike MC, Becker T, Humphreys K, Olsson M, Lie BA. Conditional analyses on the T1DGC MHC dataset: novel associations with type 1 diabetes around HLA-G and confirmation of HLA-B. Genes Immun 2009; 10: 56-67.

24 Bolognesi E, Karell K, Percopo S, Coto I, Greco L, Mantovani V et al. Additional factor in some HLA DR3/DQ2 haplotypes confers a fourfold increased genetic risk of celiac disease. Tissue Antigens 2003; 61: 308-316.

25 McDonald WI, Compston A, Edan G, Goodkin D, Hartung HP, Lublin FD et al. Recommended diagnostic criteria for multiple sclerosis: guidelines from the International Panel on the diagnosis of multiple sclerosis. Ann Neurol 2001; 50: 121-127.

26 Lublin FD, Reingold SC. Defining the clinical course of multiple sclerosis: results of an international survey. Neurology 1996; 48: 907-911.

27 Tonks S, Marsh SGE, Bunce M, Moses JH, Krausa P, Sadler AM et al. HLA class I DNA typing study. In: D Charron (ed). Genetic Diversity of HLA: Functional and Medical Implications. EDK: Sevres, 1997, pp 199-215.

28 Dudbridge F. Pedigree disequilibrium tests for multilocus haplotypes. Genet Epidemiol 2003; 25: 115-121.

29 Mattiuz PL, Ihde D, Piazza A, Ceppellini R, Bodmer WD. New approaches to the population genetics and segregation analysis of the HLA system. In: P Terasaki (ed). Histocompatibility Testing. Munksgaard: Copenhagen, 1970, pp 193-205.

This work is licensed under the Creative Commons Attribution-NonCommercial-Share Alike 3.0 Licence. To view a copy of this licence, visit http://creativecommons.org/licenses/by-nc-sa/3.0/ 